PROCEEDINGS OF THE

AMERICAN MATHEMATICAL SOCIETY

Volume 124, Number 4, April 1996

\title{
HNN BASES AND HIGH-DIMENSIONAL KNOTS
}

\author{
DANIEL S. SILVER
}

(Communicated by James West )

\begin{abstract}
There exists a 3-knot group having HNN bases of two types: bases that are arbitrarily large finitely presented and bases that are arbitrarily large finitely generated but not finitely presented. Any $n$-knot with such a group has a Seifert manifold that can be converted to a minimal one by a finite sequence of ambient 0- and 1-surgeries, but cannot be converted by 1-surgeries alone.
\end{abstract}

\section{INTRODUCTION}

Let $G$ be a group and $h: G \rightarrow \mathbf{Z}$ an epimorphism. We can describe $G$ as an HNN extension $\left\langle x, B \mid x s x^{-1}=\phi(s) \forall s \in S\right\rangle$, where $h(x)=1, B \leq \operatorname{ker} h, S \leq B$, and $\phi$ is an isomorphism $\phi: S \stackrel{\sim}{\rightarrow} T \leq B$. The group $B$ is called an $H N N$ base (or base) for $G$ relative to $h$, while $S$ and $T$ are called associated subgroups. When $G$ is finitely presented, we can find such an extension in which $B, S$, and $T$ are finitely generated $[\mathbf{3}]$.

Section 2 of this paper is concerned with the case that $G$ is an $n$-knot group. A base for an $n$-knot group will mean an HNN base relative to the abelianization homomorphism. We recall that an $n$-knot, for $n \geq 1$, is a smoothly embedded $n$-sphere $\mathcal{K} \subset \mathcal{S}^{n+2}$. The group $\pi_{1}\left(\mathcal{S}^{n+2}-\mathcal{K}\right)$ is called an $n$-knot group or the group of $\mathcal{K}$. For $n \geq 1$, any $n$-knot group is also an $(n+1)$-knot group (see [7]). A Seifert manifold for $\mathcal{K}$ is a compact, connected, oriented $(n+1)$-manifold $\mathcal{V} \subset \mathcal{S}^{n+2}$ with boundary equal to $\mathcal{K}$. By $[8]$ any $n$-knot possesses a Seifert manifold. A Seifert manifold $\mathcal{V}$ is minimal if the inclusion map $\iota$ : int $\mathcal{V} \hookrightarrow \mathcal{S}^{n+2}-\mathcal{K}$ induces a monomorphism of fundamental groups.

If an $n$-knot $\mathcal{K}$ has a minimal Seifert manifold, then the group of $\mathcal{K}$ has a finitely presented base. Such a base can be realized as the fundamental group of the compact manifold obtained by "splitting" $\mathcal{S}^{n+2}$ along the Seifert manifold [8]. This fact was used in $[\mathbf{1 3}]$ in order to produce examples of $n$-knots, for $n>2$, with no minimal Seifert manifolds. Whether or not there exists a 2-knot with no minimal Seifert manifold is an open question. (See [9] for an example of a knotted torus $\mathcal{T}$ in $\mathcal{S}^{4}$ such that $\pi_{1}\left(\mathcal{S}^{4}-\mathcal{T}\right)$ has no HNN decomposition with finitely presented base.) In higher dimensions the situation is clearer. We proved in [14] that any $n$-knot $\mathcal{K}$, for $n>2$, has a minimal Seifert manifold if and only if its group has a finitely presented base. We showed moreover that in this case any Seifert manifold for $\mathcal{K}$ can be converted to a minimal one by a finite sequence of ambient 0 - and 1-surgeries. (Ambient $i$-surgery is also called $(i+1)$-handle exchange $[\mathbf{8}]$.)

Received by the editors May 17, 1994.

1991 Mathematics Subject Classification. Primary 57Q45; Secondary 20E06, 20 F05. 
In view of the above-mentioned results it is natural to ask whether a finitely presented group can have an HNN base (relative to some $h$ ) that is finitely presented and another that is finitely generated but not finitely presented. Can an $n$-knot group have this property? We begin with a simple construction that answers the first question affirmatively.

Let $H$ be any finitely presented group with a subgroup $H_{0}$ that is finitely generated but not finitely presented. For definiteness one can use the example of J. Stallings $[\mathbf{1 6}]$ in which

$$
H=\left\langle t, a_{1}, a_{2}, b \mid t a_{1} t^{-1}=a_{1}, t a_{2} t^{-1}=a_{2}, t b t^{-1}=a_{1} b a_{1}^{-1}, a_{1} b a_{1}^{-1}=a_{2} b a_{2}^{-1}\right\rangle
$$

while $H_{0}$ is the subgroup $g p\left(a_{1}, a_{2}, b\right)$ generated by $a_{1}, a_{2}$, and $b$. Given any such pair $\left(H, H_{0}\right)$, we define

$$
G=\left\langle x, H \mid x^{2} h x^{-2}=h \forall h \in H_{0}\right\rangle .
$$

Since $H_{0}$ is finitely generated, $G$ has a finite presentation. Let $\hat{H}_{0}$ be an isomorphic copy of $H_{0}$ with isomorphism $\hat{h} \mapsto h$. Then

$$
\begin{aligned}
G & \cong\left\langle x, H * \hat{H}_{0} \mid x^{2} h x^{-2}=h \forall h \in H_{0}, \hat{h}=x^{-1} h x \forall \hat{h} \in \hat{H}_{0}\right\rangle \\
& \cong\left\langle x, H * \hat{H}_{0} \mid x h x^{-1}=\hat{h} \forall h \in H_{0}, x \hat{h} x^{-1}=h \forall \hat{h} \in \hat{H}_{0}\right\rangle .
\end{aligned}
$$

This last presentation exhibits $G$ as an HNN extension with base $B=H * \hat{H}_{0}$ relative to the homomorphism $h: G \rightarrow \mathbf{Z}$ that maps $x$ to 1 and $H * \hat{H}_{0}$ to 0 . The associated subgroups $S$ and $T$ are the same, namely $H_{0} * \hat{H}_{0}$, while $\phi: S \rightarrow T$ is the isomorphism that interchanges factors. Since $H$ and $H_{0}$ are finitely generated, so is the base $B$. However, since $H_{0}$ is not finitely presented, neither is $B$.

In order to show that $G$ has a finitely presented HNN base, we introduce some notation. For any subgroup $A \leq B$ and integer $\nu$, we let $A_{\nu}$ denote the subgroup $x^{\nu} A x^{-\nu} \leq \operatorname{ker} h$. With this notation, ker $h$ can be described as the infinite amalgamated free product of the groups $B_{\nu}, \nu \in \mathbf{Z}$, in which $S_{\nu+1}$ is identified with $T_{\nu}$ by the mapping $x^{\nu+1} s x^{-\nu-1} \mapsto x^{\nu} \phi(s) x^{-\nu} \forall s \in S$. For integers $i \leq j$ let $B_{i, j}$ denote the subgroup of ker $h$ generated by $\bigcup_{i \leq \nu \leq j} B_{\nu}$. Whenever $i<j$ we can describe $G$ as an HNN extension with base $B_{i, j}$, associated subgroups $B_{i, j-1}, B_{i+1, j}$ and isomorphism $B_{i, j-1} \rightarrow B_{i+1, j}$ described by the map $\sigma: x^{\nu} g x^{-\nu} \mapsto x^{\nu+1} g x^{-\nu-1}$. In particular, the base $B_{0,1}$ is the amalgamated free product of two copies of $H * \hat{H}_{0}$ in which $H_{0} * \hat{H}_{0}$ in the first factor is identified with $H_{0} * \hat{H}_{0}$ in the second by an isomorphism that interchanges the factors. Clearly, this base is isomorphic to $H * H$, a finitely presented group.

A byproduct of our construction is a finitely presented group $H * H$ with a nontrivial amalgamated free product decomposition in which the factors, both isomorphic to $H * \hat{H}_{0}$, and the amalgamated subgroups, isomorphic to $H_{0} * \hat{H}_{0}$, are finitely generated but not finitely presented. The first such example was given by G. Baumslag and P. Shalen in [2] using wreath products of groups. Our example is relatively elementary, and it suggests the following. 
Proposition 1.1. Let $H$ be a finitely presented group. Then $H$ has a subgroup that is finitely generated but not finitely presented if and only if $H * H$ has an amalgamated free product decomposition in which some factor or amalgamated subgroup is finitely generated but not finitely presented.

Proof. We have seen the forward implication. In order to prove the converse, assume that $H * H$ has an amalgamated free product decompostion $A *_{C} B$ in which at least one of $A, B$ or $C$ is finitely generated but not finitely presented. By the Kurosh Subgroup Theorem that subgroup is a free product of a free group and certain subgroups of conjugates of $A$ and/or $B$. Each of the factors is finitely generated, but at least one must fail to be finitely presented. Hence $H$ contains a subgroup that is finitely generated but not finitely presented.

In the construction above we showed that the base $B_{0,1}$ is finitely presented. More generally, one sees that $B_{i, j}$ is finitely presented whenever $j-i>0$. This follows also from the next result.

Proposition 1.2. Assume that $B$ is an $H N N$ base for a finitely presented group $G$. If $B$ is finitely presented, then for all integers $i<j$, the base $B_{i, j}$ is also finitely presented.

Proof. Assume that $S$ and $T$ are the associated subgroups corresponding to $B$. For any integers $i<j$, the base $B_{i, j}$ is the free product of $j-i+1$ copies of $B$ amalgamated along copies of $S$, and by [1] such a group is finitely presented if and only if $S$ is finitely generated. Associated subgroups $S, T$ of an HNN decomposition for $G$ need not be finitely generated. However, they must be finitely generated if the base $B$ is finitely presented. To see this, consider the presentation

$$
\left\langle x, B \mid x s x^{-1}=\phi(s) \forall s \in S\right\rangle \text {. }
$$

Since $G$ is finitely presented, only finitely many relators are needed [11], say those of a finite presentation of $B$ together with $x s_{1} x^{-1}=\phi\left(s_{1}\right), \ldots, x s_{n} x^{-1}=\phi\left(s_{n}\right)$. Let $\tilde{S}$ be the subgroup of $S$ generated by $s_{1}, \ldots, s_{n}$, and let $\tilde{T}=\phi(\tilde{S})$. Replacing $S$ and $T$ with their respective subgroups $\tilde{S}$ and $\tilde{T}$, known to be finitely generated, we can conclude that the base $B_{i, j}$ is finitely presented. Of course, we can also conclude that the original associated subgroups $S$ and $T$ were finitely generated, since $B_{i, j}$ cannot be both finitely presented and nonfinitely presented.

\section{High-DIMENSIONAL KNOT GROUPS}

Proposition 1.2 implies that if a finitely presented group $G$ has an HNN base that is finitely presented, then it has finitely presented bases that are "arbitrarily large" in the following sense.

Definition 2.1. A group $G$ has arbitrarily large finitely presented (resp. finitely generated but not finitely presented) HNN bases relative to $h: G \rightarrow \mathbf{Z}$ if $G$ has finitely presented (resp. finitely generated but not finitely presented) HNN bases $B(k), k=1,2 \ldots$, such that $\bigcup_{k} B(k)=\operatorname{ker} h$.

Theorem 2.2. There exists a 3-knot group $G$ with arbitrarily large finitely presented HNN bases and arbitrarily large finitely generated but not finitely presented 
HNN bases. Any n-knot with such a group has a Seifert manifold that can be converted to a minimal one by a finite sequence of ambient 0 - and 1-surgeries, but cannot be converted by ambient 1-surgeries alone.

Remark. The last statement of Theorem 2.2 says that certain Seifert manifolds can be made minimal by ambient surgery only if their fundamental groups are first enlarged by attaching "hollow 1-handles" to the Seifert manifolds.

Lemma 2.3. Let $G=\left\langle x, B \mid x s x^{-1}=\phi(s) \forall s \in S\right\rangle$ be a group with $H N N$ base $B$ relative to $h: G \rightarrow \mathbf{Z}$ and associated subgroups $S$ and $T$, and let $A$ be a subgroup of $B$. Assume that $A$ and $T$ are free factors of the subgroup that they generate. Then $x^{-1} A x$ and $B$ are free factors of $g p\left(x^{-1} A x, B\right) \leq \operatorname{ker} h$, and $g p\left(x^{-1} A x, B\right)$ is a base for $G$ relative to $h$.

Proof. Let $\hat{A}$ be an isomorphic copy of $A$ with isomorphism $\hat{a} \mapsto a$, and consider the presentation

$$
\left\langle x, \hat{A} * B \mid x \hat{a} x^{-1}=a \forall \hat{a} \in \hat{A}, x g x^{-1}=\phi(g) \forall g \in S\right\rangle .
$$

The relations $x \hat{a} x^{-1}=a$ can be rewritten as $\hat{a}=x^{-1} a x$, thereby identifying $\hat{A}$ with $x^{-1} A x \leq G$; consequently, (1.1) is a presentation for $G$. Since $g p(A, T) \cong A * T$, the mappings $\hat{a} \mapsto a \forall a \in \hat{A}$ and $g \mapsto \phi(g) \forall g \in S$ determine an isomorphism between $\hat{A} * S \leq \hat{A} * B$ and $g p(A, T)$. It follows that (1.1) presents $G$ as an HNN extension with base $\hat{A} * B$. Since $\hat{A}$ is identical to $x^{-1} A x$ in $G$, the lemma is proved.

Proof of Theorem 2.2. J. Hillman showed in $[\mathbf{6}]$ that the following presentation describes the group of a 2 -knot $\mathcal{K}$ :

$$
\left\langle x, a, b \mid a^{2}=(a b)^{3}=b^{5}, x a x^{-1}=a, x b x^{-1}=b^{-1} a^{-1} b^{2} a b\right\rangle .
$$

The commutator subgroup of this group is the binary icosahedral group $I$, a perfect group of order 120. Observe that the element $a \in I$ commutes with the meridianal generator $x$. Define $U$ to be the group of the product 2 -knot $\mathcal{K} \sharp \mathcal{K}$ with presentation

$$
\begin{aligned}
\left\langle x_{U}, a_{1}, b_{1}, a_{2}, b_{2}\right| a_{i}^{2}= & \left(a_{i} b_{i}\right)^{3}=b_{i}^{5}, x_{U} a_{i} x_{U}^{-1}=a_{i}, \\
& \left.x_{U} b_{i} x_{U}^{-1}=b_{i}^{-1} a_{i}^{-1} b_{i}^{2} a_{i} b_{i}, i=1,2\right\rangle .
\end{aligned}
$$

The commutator subgroup $U^{\prime}$ of $U$ is the free product $I * I$, a finitely generated perfect group. The element $l=a_{1} a_{2}$ has infinite order and commutes with the meridianal generator $x_{U}$. The subgroup $g p\left(l, x_{U}\right)$ is isomorphic to $\mathbf{Z} \oplus \mathbf{Z}$.

Let $V$ be the group of any 3-knot for which no minimal Seifert manifold exists. The commutator subgroup of $V$ must contain a finitely generated subgroup $W$ that is not finitely presented. In fact, arguments of $[\mathbf{1 4}, \mathrm{p}$. 106] show that $W$ can be chosen to be $\iota_{*} \pi_{1}($ int $\mathcal{V})$, where $\mathcal{V}$ is any Seifert manifold for the 3 -knot. The main idea is that if $\iota_{*} \pi_{1}($ int $\mathcal{V})$ were finitely presented, then the kernel of $\iota_{*}$ would be the normal closure of only finitely many elements of $\pi_{1}($ int $\mathcal{V})$, elements that can be represented by embedded loops in $\mathcal{V}$ and then killed by ambient 1-surgery.

Let $\tilde{U}$ be an isomorphic copy of $U$ with isomorphism $\tilde{u} \mapsto u$. Define

$$
B \cong\left\langle V * U * \tilde{U} \mid x_{V}=x_{U}=\tilde{l}, l=\tilde{x}_{\tilde{U}}\right\rangle .
$$


The group $B$ is the amalgamated free product of the 3-knot groups $\left\langle V * U \mid x_{V}=x_{U}\right\rangle$ and $\tilde{U}$ in which the free abelian subgroup generated by $x_{U}$ and $l$ in the first factor is identified with the free abelian subgroup generated by $\tilde{l}$ and $\tilde{x}_{\tilde{U}}$ in the second factor. We record the observations for later use that the subgroup of $B$ generated by $V^{\prime}$ and $U^{\prime}$ is the commutator subgroup of $\left\langle V * U \mid x_{V}=x_{U}\right\rangle$, and that this group is simply the free product of $V^{\prime}$ and $U^{\prime}$.

Clearly $H_{1} B$ is trivial. Using the Mayer-Vietoris sequence [4] (or see [16]) and the fact that every $n$-knot group has vanishing second homology [7], one checks that $B$ is superperfect; i.e., $H_{2} B$ is also trivial. Finally define

$$
G \cong\left\langle x, B \mid x g x^{-1}=x_{U} g x_{U}^{-1} \forall g \in U^{\prime}\right\rangle
$$

Since $B$ is finitely presented and $U^{\prime}$ is finitely generated, $G$ is finitely presented. Presentation (2.1) displays $G$ as an HNN extension with base $B$, associated subgroups $S, T$ equal to $U^{\prime}$, and isomorphism $\phi: S \stackrel{\sim}{\rightarrow}$ given by the meridianal automorphism $g \mapsto x_{U} g x_{U}^{-1}$ in $U$. Since $B$ is perfect, $H_{1} G$ is an infinite cyclic group generated by $x$. It is a straightforward exercise to check that $x$ normally generates $G$. One begins by killing $x$, thereby introducing the relations $g=x_{U} g x_{U}^{-1} \forall g \in U^{\prime}$ which kill $U^{\prime}$ (since $U$ is normally generated by $x_{U}$ ) thereby killing $l$, etc. Also, the exact sequence for HNN extensions [4] (or see [5]) combined with the facts that $H_{2} B=0$ and $H_{1} U^{\prime}=0$ enable one to check that $H_{2} G=0$. Hence by Kervaire's theorem [7] the group $G$ is a 3 -knot group.

Using the notation established in Section 1, we can describe $G$ as an HNN extension with base $B_{i, j}$, associated subgroups $B_{i, j-1}, B_{i+1, j}$ and isomorphism $B_{i, j-1} \rightarrow B_{i+1, j}$ determined by $\sigma: x^{\nu} g x^{-\nu} \mapsto x^{\nu+1} g x^{-\nu-1}$. In this way we obtain arbitrarily large finitely presented bases $B(k)=B_{-k, k}, k>0$, for $G$.

We now produce arbitrarily large bases $B^{*}(k)$ for $G$ that are finitely generated by not finitely presented. Recall that $V^{\prime}$ contains a subgroup $W$ that is finitely generated but not finitely presented. For any integers $i<j$, the base $B_{i-1, j}$ is the amalgamated free product of $B_{i-1}$ and $B_{i, j}$ in which $U_{i-1}^{\prime}$ is identified with $U_{i}^{\prime}$. Earlier we observed that $g p\left(V^{\prime}, U^{\prime}\right)$ is the free product $V^{\prime} * U^{\prime}$. Hence $g p\left(W, U^{\prime}\right)$ is $W * U^{\prime}$. It follows that the subgroup of $B_{i-1, j}$ generated by $W_{i-1}, U_{i-1}^{\prime}$, and $B_{i, j}$ is the amalgamated free product of $W_{i-1} * U_{i-1}^{\prime}$ and $B_{i, j}$ in which $U_{i-1}^{\prime}$ is identified with $U_{i}^{\prime} \leq B_{i, j}$, and this group is clearly $W_{i-1} * B_{i, j}$. Hence $W_{i-1}$ and $B_{i, j}$ are free factors of the subgroup of $B_{i-1, j}$ that they generate. By Lemma 2.3 the subgroup $W_{i-1} * B_{i, j}$ is a base $B_{i, j}^{*}$ for $G$. Of course, $B_{i, j}^{*}$ is finitely generated since each of its factors is, but it is not finitely presented since $W_{i-1}$ is not. For $k>0$, we define $B^{*}(k)$ to be $W_{-k-1} * B_{-k, k}$. Then $\bigcup_{k} B^{*}(k)$ is equal to $G^{\prime}$, and the first statement of Theorem 2.2 is proved.

Assume that $\mathcal{K}$ is any 3-knot with group $G$, and let $\mathcal{V}$ be a Seifert manifold for $\mathcal{K}$. The image $\iota_{*} \pi_{1}($ int $\mathcal{V})$ in $\pi_{1}\left(\mathcal{S}^{3}-\mathcal{K}\right)$ is a finitely generated subgroup of $G^{\prime}$. Choosing $k>0$ sufficiently large, we can assume that the image is contained in the base $B^{*}(k)$, and since $B^{*}(k)$ is finitely generated, we can perform ambient 0 -surgeries on $\mathcal{V}$ until the image coincides with $B^{*}(k)$ (see [14] pp. $\left.106-107\right)$. The new Seifert manifold $\mathcal{V}^{\prime}$ that we obtain cannot be converted to a minimal one by any finite sequence of ambient 1 - surgeries alone because $\iota_{*} \pi_{1}\left(\right.$ int $\left.\mathcal{V}^{\prime}\right)$ is not finitely related. 


\section{A CONJECTURE ABOUt KNOT-LIKE GROUPS}

Following Rapaport [12] we will say that a group $G$ is knot-like if $G / G^{\prime} \cong \mathbf{Z}$ and $G$ has a finite presentation in which the number of generators exceeds the number of relators by one. Any 1-knot group is a knot-like group as are the groups of many $n$-knots for $n>1$. By a base for a knot-like group we will mean an HNN base with respect to the abelianization homomorphism. By [3] every knot-like group has a finitely generated base.

Conjecture 3.1. Every finitely generated base for a knot-like group is finitely presented.

It is well known and not difficult to prove that if the commutator subgroup $G^{\prime}$ of a knot-like group is finitely generated, then any finitely generated base for $G$ is equal to $G^{\prime}$. A conjecture of Rapaport $[\mathbf{1 2}]$ asserts that in this case any finitely generated base for $G$ is in fact a free group. Partial results about this conjecture were obtained in $[\mathbf{1 2}]$ and $[\mathbf{1 5}]$ (see also $[\mathbf{1 0}]$ ).

\section{REFERENCES}

1. G. Baumslag, A remark on generalized free products, Proc. Amer. Math. Soc. 13 (1962), 53-54. MR 26:202

2. G. Baumslag, P.B. Shalen, Amalgamated products and finitely presented groups, Comment. Math. Helv. 65 (1990), 243-254. MR 91j:20071

3. R. Bieri, R. Strebel, Almost finitely presented soluble groups, Comment. Math. Helv. 53 (1978), 258-278. MR 58:16890

4. I.M. Chiswell, Exact sequences associated with a graph of groups, J. Pure Appl. Alg. 8 (1976), 63-74. MR 53:3147

5. C.McA. Gordon, Homology of groups of surfaces in the 4-sphere, Math. Proc. Camb. Phil. Soc. 89 (1981), 113-117. MR 83d:57016

6. J. Hillman, High dimensional knot groups which are not two-knot groups, Bull. Austral. Math. Soc. 16 (1977), 449-462. MR 58:31098

7. M. Kervaire, On higher dimensional knots, Differential and Combinatorial Topology (A Symposium in Honor of Marston Morse) (S.S. Cairns, ed.), Princeton University Press, Princeton, 1965, pp. 105-109. MR 31:2732

8. J. Levine, Unknotting spheres in codimension two, Topology 4 (1965), 9-16. MR 31:4045

9. T. Maeda, Knotted surfaces in the 4-sphere with no minimal Seifert manifolds, preprint.

10. D.I. Moldavanskii, Certain subgroups of groups with one defining relation (in Russian), Sibirsk. Math. Z. 8 (1967), 1370-1384. MR 36:3862

11. B.H. Neumann, Some remarks on infinite groups, J. London Math. Soc. 12 (1937), 4-11.

12. E.S. Rapaport, Knot-like groups, Annals of Math. Studies, vol. 84, Princeton Univ. Press, Princeton, 1975, pp. 119-133.

13. D.S. Silver, Examples of 3-knots with no minimal Seifert manifolds, Math. Proc. Camb. Phil. Soc. 110 (1991), 417-420. MR 92f:57030

14. D.S. Silver, On the existence of minimal Seifert manifolds, Math. Proc. Camb. Phil. Soc. 114 (1993), 103-109. MR 94c:47039

15. D.S. Silver, On knot-like groups and ribbon concordance, J. Pure Appl. Alg. 82 (1992 99-105). MR 94a:57021

16. J. Stallings, A finitely presented group whose 3-dimensional integral homology is not finitely generated, Amer. Journal of Math. 95 (1963), 541-543. MR 28:2139

Department of Mathematics and Statistics, University of South Alabama, Mobile, Alabama 36688

E-mail address: silver@mathstat.usouthal.edu 